\title{
La Corse, une expérience de décentralisation originale pour la France
}

\author{
André Torre ${ }^{1}$ \\ Institut national de la recherche agronomique
}

La France n'a pas une grande habitude, ni une grande expérience, des politiques de décentralisation et se distingue nettement sur ce point de ses plus proches voisins : l'Allemagne (avec les Landers), l'Italie (qui a accordé une autonomie importante à de nombreuses régions, dont la Sicile et la Sardaigne) ou l'Espagne (avec les Canaries ou les Baléares). Au contraire, l'État français possède une tradition centralisatrice bien établie qui constitue même un des socles de la République. La loi fondamentale affirme les principes d'unité et d'indivisibilité de la République. L'unification des langues, des coutumes et des économies locales a constitué l'une des constantes de la République française, en particulier avec les mesures prises par les pères de l'école publique qui a longtemps constitué le vecteur principal de la propagation d'une norme de pensée et de comportement au niveau national, ainsi que de la diffusion de la langue française jusqu'au fin fond des zones les plus reculées du territoire. Cette attitude est d'autant plus compréhensible que le territoire français s'est constitué par adjonctions successives et que ses frontières sont longtemps restées mouvantes, y compris à la fin du XIX ${ }^{\mathrm{e}}$ siècle qui a vu, par exemple, le retour de l'Alsace et de la Lorraine dans le giron allemand.

Pourtant, la nécessité d'une politique d'aménagement du territoire a commencé à se faire sentir aux pouvoirs publics dès les lendemains de la Seconde Guerre mondiale. Avec la planification apparaît un souci pour l'aménagement, mais c'est surtout dans les années 1960 que se fait jour une véritable préoc- cupation pour les questions de décentralisation, avec la prise de conscience que la richesse nationale se concentre dans la région parisienne : c'est l'époque de «Paris et du désert français », pour reprendre le titre d'un célèbre ouvrage de J.F. Gravier.

Cette préoccupation, De Gaulle sera l'un des premiers hommes politiques à la défendre et à vouloir la mettre en application. Mal lui en prend: c'est en 1969 qu'il démissionne de la présidence de la République à la suite de l'échec du référendum en faveur de la décentralisation dans lequel il s'était totalement engagé. Il n'a pas été suivi par sa majorité, et en particulier par les grands dirigeants des partis au pouvoir, dans sa vision décentralisatrice. L'héritage jacobin sur lequel s'est construit la France contemporaine est resté bien vivant. Les tentatives de décentralisation vont alors rester modestes, à l'exception de la Loi Deferre de 1982 qui attribue le statut de collectivités locales aux régions, ce qui signifie une élection au suffrage universel et des compétences élargies. Les transferts de compétence et de souveraineté restent limités. Pourtant, aujourd'hui, la question de la décentralisation revient dans les débats et le gouvernement Raffarin a entrepris la mise en œuvre d'une nouvelle politique d'acquisition de compétences par les régions françaises.

Dans l'ensemble des 22 régions françaises encore dépositaires de peu de compétences, la Corse constitue un cas tout à fait intéressant en matière de décentralisation. Si elle s'est longtemps caractérisée par des conflits de souveraineté et une tendance massive à la désertification, la réapparition plus récente 
d'une revendication autonomiste, puis nationaliste, les conflits qui s'en sont suivis et les avancées en matière de décentralisation et de statuts régionaux ont fait de la région corse un véritable laboratoire d'expériences avec ses échecs (souvent éclatants), ses avancées (souvent modestes) et ses succès (parfois bien réels, comme dans les cas de la viticulture ou du développement des activités culturelles). C'est à ce titre qu'il faut comprendre la place importante qu'elle occupe dans les textes législatifs ainsi que dans les médias, qui parlent volontiers de «question corse ", plus que par ses performances économique (la plus faible production des régions françaises) ou démographique (la plus petite population) qui restent extrêmement modestes.

\section{La revendication nationaliste a toujours été présente en Corse, et les mouvements en faveur d'une autonomie n'ont jamais cessé leur activité de résistance.}

Dès le début, les relations entre la Corse et la France ne sont pas simples : achetée à la République de Gènes en 1768, la Corse a fait jusqu'alors essentiellement l'objet d'une colonisation et d'échanges commerciaux sur ses côtes, alors que le centre de l'île, très montagneux et donc difficilement accessible, est géré par les clans locaux et vit d'une économie agricole organisée autour de la culture de la châtaigne. Pour autant, les élites sont fortement italianisées, et il est de bon ton de faire ses études à Rome ou à Gènes (les langues italienne et corse étant assez proches), puis de servir dans les armées des principautés italiennes ou du Pape. L'arrivée des Français, qui ont une conception de leur mission colonisatrice bien plus étendue que celle de Gènes (limitée au commerce), va se faire dans la violence. Les Corses refusent cette domination, s'organisent, lèvent une armée et livrent une guerre aux bataillons français, guerre qui se termine par une défaite finale et la reddition des troupes corses.

S'ensuit une période d'installation lente de la puissance française qui se trouve rapidement confrontée par ailleurs à d'autres problèmes, en particulier avec la Révolution française. Les élites corses se partagent entre l'Italie (plus proche géographiquement) et la
France, apprennent à parler cette nouvelle langue et trouvent leur chemin à Paris. Le meilleur exemple en est Napoléon qui, devenu Empereur de France, n'accordera pas d'attentions spécifiques à sa Corse natale, en bon héritier politique des jacobins.

L'État français va progressivement installer ses structures dans toute la Corse, qui devient un Département français, et imposer, avec beaucoup de difficultés et de mesures de rétorsion la langue française. Les Corses, majoritairement ruraux et exploitant des terres peu fertiles, vont continuer à s'expatrier, d'abord en Amérique du Nord ou du Sud, puis dans les colonies françaises, jusqu'à la Seconde Guerre mondiale. Ils fournissent un gros contingent de douaniers, de militaires, de fonctionnaires et d'administrateurs, et reviennent au pays pour prendre leur retraite. Ceux qui restent en Corse vivent difficilement, un peu oubliés des pouvoirs publics nationaux et bénéficiant de l'argent envoyé par la diaspora. Le choc majeur va provenir de la Première Guerre mondiale qui décime l'essentiel des hommes (il suffit de regarder les monuments funéraires dans les villages corses). À titre de récompense de cet engagement, les survivants bénéficient d'emplois réservés dans l'administration française et quittent l'île. Il s'ensuit une désertification massive qui se produit beaucoup plus tôt que dans les autres régions françaises. La Corse s'endort et rate le coche de l'industrialisation...

Dépeuplée, sous-équipée, difficile d'accès, économiquement sous-développée, la Corse ne bénéficie pas de beaucoup d'atouts après la Seconde Guerre mondiale pendant laquelle elle est redevenue une province italienne suite à l'invasion des troupes de Mussolini. C'est, de manière indirecte, la Guerre d'Algérie qui va lui redonner une nouvelle énergie. L'indépendance de l'Algérie, en 1962, en chasse les colons qui avaient fait fortune en cultivant la vigne et en vendant leur vin en France : naît alors l'idée de transplanter en Corse la viticulture caractéristique des plaines de la Mitidja. Les pouvoirs publics accordent aux colons venus d'Algérie des avantages exorbitants en matière d'accession à la propriété, de prêts et de mise en valeur des terres, des avantages qui sont refusés aux résidents auxquels on a préalablement racheté ces terres à faible prix. Le 
mécontentement devient important dans la population locale et entraîne une renaissance de la revendication autonomiste. Elle va connaitre un premier épisode sanglant en 1975, alors que l'implantation d'une viticulture de masse de type colonial se révèle déjà un échec, avec l'occupation d'une cave viticole de colons par des militants. Les policiers vont payer de leur vie l'assaut donné aux insurgés.

La revendication nationaliste a toujours été présente en Corse, et les mouvements en faveur d'une autonomie n'ont jamais cessé leur activité de résistance, passant de l'affirmation d'une identité propre à la recherche de liens privilégiés avec l'Italie. Très minoritaire, elle se réactive dans les années 1960 sous l'impulsion de l'arrivée des colons, mais également à une période de renaissance des luttes de libération nationales. Les années 1970 voient l'apparition de mouvements autonomistes puis nationalistes qui ne tardent pas à recourir à la force pour affirmer leurs revendications: plasticages spectaculaires en Corse et sur le Continent, enlèvements, menaces à l'encontre des non-Corses sont le lot de mouvements qui ne seront pourtant jamais aussi extrémistes que ceux du Pays Basque ou d'Irlande et répugneront généralement à faire couler le sang. À côté de ces mouvements apparaît, dans la société civile, la revendication d'une réappropriation des racines: reconnaissance et enseignement de la langue corse, réévaluation du patrimoine culturel, en particulier musical, retour au pays, volonté de reconquête de l'intérieur $\mathrm{du}$ territoire. La traduction politique en sera l'affirmation d'une identité locale, la demande de reconnaissance des spécificités de la Corse et l'affirmation de la légitimité d'un rattrapage historique important en matière d'infrastructures.

\section{Les années 1970 voient l'apparition de mouvements autonomistes puis nationalistes qui ne tardent pas à recourir à la force pour affirmer leurs revendications.}

La réponse des pouvoirs publics à cette revendication va se faire en plusieurs temps. Au début des années 1960, la Corse est généralement considérée comme une zone sans grand intérêt dont les rivages sont seulement débarrassés de la malaria depuis le passage des Américains en 1943. On envisage au mieux d'en faire une colonie de peuplement afin de combattre l'indolence bien connue de la population locale. Après la Guerre d'Algérie et l'arrivée des colons, l'intérêt augmente, avec l'espoir de la mise en place d'une viticulture de masse : hélas, le recul de la consommation de vin de table, les faibles performances des terres et le refus des autochtones d'adhérer à ce modèle ruinent bien vite cette aspiration et acculent les nouveaux arrivants à la faillite, souvent frauduleuse. Le nouveau contexte politique et social puis la montée des postures identitaires vont toutefois conduire à une série d'évolutions institutionnelles qui feront passer la Corse, en quelques années, du rang de simple Département à celui de région pilote en matière de décentralisation et d'expérimentation des nouveaux statuts.

La première étape est la création de la région corse. En effet, jusqu'en 1972, la Corse est toujours rattachée à la région PACA (Provence Alpes Côte d'Azur, qui comprend en particulier les villes de Marseille et de Nice). Elle obtient alors un statut de « région programme» et devient la $23^{\mathrm{e}}$ Région française. La seconde étape consiste en l'instauration de la bi-départementalisation en 1975. Sont ainsi tout d'abord créés deux Départements, respectivement nommés Haute Corse (préfecture : Bastia) et Corse du Sud (préfecture: Ajaccio), ce qui entraîne un dédoublement des services de l'État concernant l'agriculture, les routes, les logements ou tout simplement l'accueil des personnes. C'est le signe d'un premier accroissement du nombre de fonctionnaires, dont les salaires vont bientôt constituer la première source de revenus de l'île. L'étape suivante se produit en 1980. Après quelques années marquées par une intensification des luttes en faveur de l'autonomie, la création d'un mouvement clandestin de libération (le Front de libération nationale de la Corse) et un accroissement des attentats contre les intérêts de l'État français et des particuliers dont les installations menacent le littoral, une vieille revendication est gagnée : la création d'une université qui est installée au cœur historique de la Corse, à Corte, et fait entrer la région corse dans le droit commun. Durant la même période, les plans d'aménagement et de développement de l'île, qui font suite à une 
kyrielle de rapports plus ou moins circonstanciés, se succèdent avec des succès très mitigés.

Ces évolutions se font dans un strict cadre constitutionnel et marquent simplement un début de reconnaissance d'une région un peu oubliée jusque là par Paris. Il en va tout autrement des évolutions institutionnelles ultérieures, qui vont sortir la Corse du droit commun et constituer des innovations institutionnelles incontestables dans le paysage français. Elles se feront en trois temps, respectivement en 1982, 1991 et 2001. En effet, la situation en Corse, et les relations entre la Corse et les pouvoirs publics (qui ont autorisé en 1974 l'enseignement de la langue corse), continuent à se dégrader tout au long des années 1970, marquées par une entrée croissante de leaders autonomistes dans la clandestinité et par une radicalisation des mots d'ordre de lutte. À l'arrivée au pouvoir de François Mitterrand, en 1981, un statut particulier est promis et sera accordé en 1982. La région corse bénéficie alors de pouvoirs élargis et la première Assemblée de Corse est élue au suffrage universel en août 1982. En 1988 est défini un deuxième statut, dit «statut Joxe», du nom du ministre qui l'a négocié. En 2000, à l'instigation de Lionel Jospin, un troisième statut, connu sous le nom de "processus de Matignon », est mis en place et voté en décembre 2001 par l'Assemblée nationale.

\section{En 1980, une vieille revendication est gagnée : la création d'une université qui est installée au cœur historique de la Corse, à Corte, et fait entrer la région corse dans le droit commun.}

Le statut de 1982 sort la Corse du régime administratif des régions et la transforme en Collectivité territoriale. Elle est dotée d'une Assemblée de 61 conseillers élus au suffrage universel qui désignent un Président de région et ont la possibilité, comme les Départements d'outre-mer, de saisir le premier ministre de propositions de modification ou d'adaptation des dispositions législatives ou réglementaires. La Collectivité se voit attribuer des compétences élargies concernant les domaines de l'éducation et de la formation, de la communication, de la culture et de l'environnement, de l'aménagement et de l'urba-

nisme, de l'agriculture, du logement, des transports, de l'emploi et de l'énergie; elle est également munie de différents Offices, qui sont des établissements publics en charge du développement agricole et rural, des questions hydraulique et des transports, avec des moyens propres et des possibilités de recrutement. La Corse est à l'époque la seule région française à disposer d'un pareil dispositif, mais elle est rapidement rattrapée par le statut commun des autres régions françaises, en particulier en matière électorale ou de transfert des compétences.

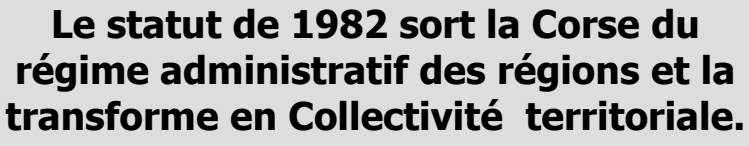

Il faut attendre 1991, avec le statut Joxe, dont les caractéristiques rappellent celles mises en place pour la Polynésie en 1984, pour assister à de nouvelles avancées institutionnelles. Les années 1980 sont marquées par l'entrée des nationalistes dans la vie politique : ils participent aux élections et deviennent une force qui va représenter jusqu'à $25 \%$ des électeurs, sans que la violence connaisse de ralentissement notable. Le statut de 1991, qui vise à redonner à la Corse une «avance » institutionnelle et à la conforter dans sa spécificité, la dote d'une organisation inédite et restée à ce jour sans équivalent en France métropolitaine. La nouvelle constitution, de type parlementaire, se caractérise avant tout par la création d'un exécutif doté d'un président et de six conseillers responsables devant l'Assemblée et couvrant les domaines économiques, sociaux et culturels. Cet exécutif, issu de la majorité régionale, est nommé par le président de la Collectivité et joue un peu le même rôle que le Premier ministre et son gouvernement auprès du président de la République, en France. Les Offices, maintenant présidés par les conseillers, sont maintenus, et même augmentés par la création d'un Office de l'environnement, mais la principale avancée au plan économique réside dans la création d'une Agence de développement économique qui a pour mission d'aider et de planifier le développement local. Les nationalistes et les autonomistes restent toutefois peu satisfaits de ce nouveau statut, car l'avancée la plus fondamentale, ou du moins la plus attendue, proposée par le 
gouvernement et votée par l'Assemblée nationale, est refusée par le Conseil constitutionnel qui la juge contraire à la Constitution de la France : il s'agit de la notion de peuple corse (composante du peuple français).

Les années 1990 sont des années de plomb, marquées par la lutte fratricide entre les différentes factions nationalistes, par l'assassinat du préfet Erignac en 1998 et par la difficulté à trouver des solutions pour la Corse. Le régime de la Collectivité se met lentement en place avec le recrutement de nombreux nouveaux fonctionnaires territoriaux, sans que la situation économique de l'île connaisse d'amélioration spectaculaire. À la suite d'une grève générale qui dure plusieurs mois, le gouvernement Juppé décide, en 1996, de la création d'une zone franche pour l'ensemble de la région corse. Elle se caractérise en particulier par une exonération totale d'impôts sur les bénéfices pour les sociétés pour une période de cinq années.

\section{On peut retenir trois grandes phases d'évolution, mais également de résistance aux changements de nature institutionnelle proposés par les différents gouvernements.}

Les années 2000 semblent d'ores et déjà plus fertiles en événements, qu'ils soient provoqués par ces contingences locales ou qu'ils résultent des évolutions institutionnelles en marche au niveau national. On peut retenir trois grandes phases d'évolution, mais également de résistance aux changements de nature institutionnelle proposés par les différents gouvernements.

- La première phase concerne ce qu'on appelle couramment le processus de Matignon, opération initiée sous la responsabilité de Lionel Jospin à partir de 2000. Devant le caractère instable de la situation politique et économique, des rencontres ont été initiées, qui associent les représentants élus des différentes sensibilités politiques de la région corse (dont les nationalistes) et les représentants de l'État. Ce processus, dont on attendait des résultats importants, a certainement déçu dans sa conclusion. Mais il a eu le mérite de faire dialoguer les élus (et la population) corses et de leur faire prendre des positions communes en matière de développement, la question économique devenant maintenant centrale. Les solutions proposées par l'Assemblée de Corse n'ont pas toutes été acceptées, loin s'en faut, comme le montrent les exemples d'une possibilité de délégation législative ou du caractère obligatoire de l'enseignement de la langue corse, mais pour la première fois s'est exposée une vision commune du développement à venir, refusant en particulier une option de tourisme de masse et demandant une protection accrue du littoral. Le nouveau statut de janvier 2002, qui s'en est suivi, prévoyait une suppression des deux Départements au profit d'un mandat plus clair de la Collectivité, qui bénéficie maintenant d'un pouvoir réglementaire limité, ainsi qu'une décentralisation des nouvelles compétences dans les domaines de l'aménagement du territoire, de la protection du patrimoine et du développement économique. Les avancées étaient censées se faire en deux étapes : la première, modeste, en 2002, la seconde, beaucoup plus importante, en 2004, avec un nouveau passage devant l'Assemblée nationale.

\section{Les électeurs refusent, lors du scrutin du 6 Juillet 2003, la poursuite du processus d'autonomisation de la région corse, à une faible majorité.}

- L'arrivée de la Droite au pouvoir à la suite de la réélection de Jacques Chirac à la Présidence de la République en avril 2002 est venue changer quelque peu cette donne avec la volonté clairement affichée du gouvernement Raffarin de relancer le processus de décentralisation au niveau national. Cette volonté s'est traduite, après la tenue d'états généraux de la décentralisation, par la Loi constitutionnelle du 28 mars 2003 relative à l'organisation décentralisée de la République qui introduit un changement fondamental dans la Constitution française : si la France est toujours une République indivisible, laïque, démocratique et sociale, il apparaît dorénavant que « son organisation est décentralisée », ces avancées institutionnelles bénéficiant à l'ensemble des régions 
françaises. En particulier, le caractère déterminant des Collectivités territoriales est affirmé (communes, départements, régions, collectivités à statut particulier et collectivités d'outre-mer), ainsi que la possibilité pour certaines d'entre elles (les régions) de procéder à des expérimentations institutionnelles qui seront étendues à l'ensemble de la communauté nationale si elles sont jugées concluantes. Par ailleurs, sont envisagés des transferts de compétences entre l'État et les Collectivités territoriales ainsi qu'un système de péréquation destiné à éviter que la décentralisation ne se fasse au détriment des régions les moins bien dotées. Enfin, la possibilité d'un référendum régional est inscrite dans la Constitution, les électeurs pouvant être consultés sur des questions relatives à l'avenir de leur région. C'est un nouvel acte de la décentralisation qui se met en place, longtemps après les lois de 1982.

- La troisième phase consiste en une proposition de nouvelle évolution institutionnelle pour la Corse, qui va être refusée par les Corses eux-mêmes. Le ministre de l'Intérieur, Nicolas Sarkozy, partisan de la décentralisation, reprend le dossier où l'avait laissé le gouvernement Jospin et, en dépit des résistances d'une partie de la majorité, conduit de nouvelles discussions avec les élus locaux qui l'amènent à proposer la tenue d'un référendum, conformément à la nouvelle loi de mars 2003. Les électeurs corses sont appelés à voter sur une nouvelle réforme de l'organisation institutionnelle de la Corse devant conduire à la suppression des deux Départements au bénéfice de la Collectivité unique, qui assurera les compétences dévolues aux trois assemblées. Pour ne pas trop froisser les susceptibilités des élus départementaux, on a cependant conservé deux conseils territoriaux, correspondant aux anciens conseils départementaux, mais dont les attributions seront plus faibles, puisque la Collectivité conservera la haute main sur le régime des aides aux entreprises et l'élaboration du plan d'aménagement et de développement durable de la région. Ces précautions vont se révéler inutiles, tout autant que les efforts du gouvernement ou du Président de la République en faveur du oui, qui bénéficie également du soutien des principaux partis politiques nationaux ainsi que des mouvements nationalistes corses. Les électeurs, peut-être troublés par une évolution institutionnelle complexe (on supprime les deux Départements après les avoir dédoublés quelques années auparavant), refusent, lors du scrutin du 6 Juillet 2003, la poursuite du processus d'autonomisation de la région corse, à une faible majorité (51\% de non contre $49 \%$ de oui).

Ainsi, voici donc la Corse repartie dans ses évolutions complexes et parfois contradictoires, alors que le processus de décentralisation amorcé au niveau national s'engage de manière peu favorable: à l'échec du référendum dans l'île, il faut ajouter le récent recul du gouvernement sur le transfert des compétences aux régions dans l'éducation nationale. La France reste un pays difficile à réformer, profondément jacobin, et les Corses, qui ont fini par prendre des habitudes bien françaises, restent eux aussi méfiants quant aux possibilités de décentralisation...

\section{Note}

1 André Torre est directeur de recherches en économie à l'INRA (Institut national de la recherche agronomique). Son thème principal de recherche concerne l'analyse des relations de proximité entre acteurs économiques et sociaux, des relations qu'il étudie soit sous l'angle des échanges de technologie entre firmes innovantes, soit à partir de l'analyse des conflits autour des usages différents de l'espace et de la terre. 